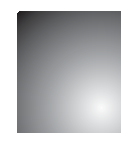

\title{
0 Papel da Confiança na Relação entre Responsabilidade Social Corporativa e o Valor de MarCA
}

\author{
The Role of Trust in the Relation Between Corporate Social \\ Responsibility and Brand Equity
}

\section{Ivan Lapuente Garrido}

Professor do PPG Administração. Universidade do Vale do Rio dos Sinos (UNISINOS). Porto Alegre, RS. Brasil. E-mail: igarrido@ unisinos.br

Fernando Rafael Cunha

Professor da Universidade Federal de Rio Grande (FURG). Rio Grande, RS. Brasil. E-mail: fernandocunha@furg.br

Fernanda Matte Cavalcante

Mestranda da Universidade do Vale do Rio dos Sinos (UNISINOS). São Paulo, SP. Brasil. E-mail: fematte1@hotmail.com

\section{Resumo}

O objetivo deste estudo é compreender o papel da confiança na relação entre a responsabilidade social corporativa (RSC) e o valor de marca (VM). Com base na literatura, foram propostos três modelos alternativos desse relacionamento. Os modelos foram testados por meio de modelagem de equações estruturais com dados coletados de uma amostra de 148 clientes de uma das maiores instituições financeiras nacionais. A partir da comparação dos modelos propostos, verificou-se a falta de suporte para a relação direta entre a RSC e o valor de marca (H1 não suportada). As hipóteses de que a confiança media a relação RSC - VM (H2) e de que essa mediação é completa $(\mathrm{H} 2 a)$ foram suportadas. Concluise que as ações de RSC contribuem para aumentar a confiança do consumidor na empresa, o que por sua vez impacta positivamente no valor de marca da instituição.

Palauras-chave: Responsabilidade Social Corporativa. Confiança. Valor de Marca. Bancos.

\begin{abstract}
The aim of this study is to comprehend the role of trust on the relation between corporate social responsibility (CSR) and brand equity (BE). Based on the literature, three different models have been developed proposing the relationship among the constructs. The models have been evaluated using Structural Equation Modeling. We have used a sample with 148 clients of one of the largest Brazilian banks. From the comparison of the proposed models was verified the absence of support for the direct relation between CSR and brand equity (H1 was not supported). The hypothesis that trust mediates the relationship between $\mathrm{CSR}-\mathrm{BE}(\mathrm{H} 2)$ and it is a complete mediation (H2a), were supported. We conclude that actions in CSR result in benefits to the organization (brand equity) through the growing of trust, or in other words, trust mediates completely the relationship between CSR and brand equity.
\end{abstract}

Keywords: Corporate Social Responsibility. Trust. Brand Equity. Banks. 


\section{INTRODUÇÃO}

A Responsabilidade Social Corporativa (RSC) tem sido tema de destaque tanto nos meios empresariais, quanto nos acadêmicos (NAN; HEO, 2007; GOLOB et al., 2008; SWAEN; CHUMPITAZ, 2008; DU et al., 2011; MANDHACHITARA; POOLTHONG, 2011, MELO; GALAN, 2011). O crescente interesse pela RSC é creditado ao reconhecimento da interdependência entre as ações das empresas e a sociedade. (GALAN; MELO, 2011; DAMEDA, 2012)

Resultados de pesquisas indicam que grupos de stakeholders, como consumidores, funcionários e investidores estão, cada vez mais, agindo de forma a beneficiar empresas socialmente responsáveis ao mesmo tempo em que procuram punir àquelas que não agem de maneira adequada para com a sociedade (PIVATO et al., 2008; NILSSON, 2009; DU et al., 2010). Enquanto as empresas socialmente responsáveis possuem melhor reputação junto a diferentes grupos de stakeholders, as empresas consideradas socialmente irresponsáveis sofrem boicotes e comunicações negativas por parte destes grupos. (OGRIZEK, 2002)

Os governos também guardam interesse nas ações sociais das empresas. Para Du et al. (2011), políticas públicas devem incentivar as ações de RSC das empresas privadas. De acordo com os autores, as áreas prioritárias para a ação social das empresas devem ser àquelas relacionadas com a sua atividade fim onde, consequentemente, detêm maior expertise. Em áreas onde não possuem expertise, as empresas podem buscar parcerias com outras organizações que são referência na atuação na área desejada (HOEFFLER; KELLER, 2002). Importante destacar que esta parceria pode ocasionar nos stakeholders efeitos de transferência entre as organizações envolvidas, ou seja, associações favoráveis que os stakeholders têm para com uma organização são transferidas também para a organização parceira. (HOEFFLER; KELLER, 2002)

A RSC pode ser vista sob duas perspectivas: altruística ou estratégica. Segundo a perspectiva altruística, as organizações se engajam em atividades sociais com o objetivo de trazer melhorias para a sociedade (ELLEN et al., 2006; PELOZA; SHANG, 2011). A perspectiva estratégica considera que as ações sociais são uma forma de melhoria da imagem corporativa, que no longo prazo se traduz em benefícios financeiros para as empresas (LUO; BHATTACHARYA, 2006; NAN; HEO, 2007; Du et al., 2011; MANDHACHITARA; POOLTHONG, 2011). Segundo a perspectiva estratégica, haveria um nível ótimo de investimento em RSC que maximizaria os lucros da organização. (MELO; GALAN, 2011)

Na visão dos stakeholders, as empresas executam ações sociais por motivos extrínsecos ou intrínsecos. As motivações extrínsecas são vistas pelos stakeholders como aquelas nas quais a empresa atua socialmente, com objetivos de aumento de lucro. Por outro lado, as motivações intrínsecas seriam consideradas pelos stakeholders como uma preocupação genuína da empresa para com o objeto da ação social. (DU et al., 2010)

Apesar da discordância em relação às motivações subjacentes às ações sociais desenvolvidas pelas empresas, os objetivos sociais e empresariais não devem ser vistos como antagônicos ou conflitantes (NILSSON, 2009; MELO; GALAN, 2011; STANALAND et al., 2011). Para que os investimentos sociais ocorram no longo prazo, é necessário que a organização apresente desempenho financeiro suficiente (ELLEN et al., 2006). Nessa linha de raciocínio, Du et al. (2010) defendem que as ações de RSC podem caracterizar um jogo de soma positiva, onde todos os atores envolvidos (empresas e sociedade) obtém ganhos.

A linha de pesquisa que considera o papel estratégico da RSC tem indicado uma série de benefícios obtidos, direta ou indiretamente, pela vinculação de empresas com causas sociais: melhor atitude do consumidor para com a empresa (NAN; HEO, 2007), reputação (STANALAND et al., 2011), intenção de compra (ELLEN et al., 2006; VLACHOS et al., 2009; DU et al., 2011), satisfação (LUO; BHATTACHARYA, 2006; SWAEN; CHUMPITAZ, 2008), lealdade (LICHTENSTEIN et al., 2004; PIVATO et al., 2008; MANDHACHITARA; POOLTHONG, 2011; STANALAND et al., 2011), recomendações (VLACHOS et al., 2009; DU et al., 2011), valor de marca (ANDRADE et al., 2010; MELO; GALAN, 2011) entre outros benefícios.

Estudos de comportamento do consumidor têm divergido a respeito da relação entre ações de RSC e suas consequências (benefícios) para as empresas. Para diversos autores (LICHTENSTEIN et al., 2004; LUO; BHATTACHARYA, 2006; DU et al., 2011; MANDHACHITARA; POOLTHONG, 2011; BENEKE et al., 
2012), a RSC influencia diretamente nas respostas dos consumidores. Porém, para outros pesquisadores (PIVATO et al., 2008; SWAEN; CHUMPITAZ, 2008; VLACHOS et al., 2009; POOLTHONG; MANDHACHITARA, 2011; STANALAND et al., 2011), as respostas dos consumidores às ações de RSC seriam parcialmente ou completamente mediadas pela confiança na organização. Em outras palavras, os benefícios para a organização só ocorreriam quando as ações de RSC impactassem positivamente a confiança dos consumidores na empresa.

Reforçando a vertente que considera a confiança um aspecto fundamental em estudos de RSC, Ellen et al. (2006) argumentam que a desconfiança em relação às empresas, de um modo geral, mostra-se como uma barreira a ser superada para que estas, efetivamente obtenham os resultados desejados de suas ações de RSC. Em relação ao setor bancário, campo de pesquisa deste trabalho, a desconfiança revela-se ainda maior do que a aferida em outros segmentos empresariais (MATTILA et al., 2010; BENEKE et al., 2012). Segundo Ogrizek (2002), a desconfiança nas instituições bancárias ocorre em função da percepção dos stakeholders de que muitos dos problemas sociais da atualidade são causados por organizações deste setor. Dessa forma, as ações de RSC dos bancos poderiam ser um meio de minimizar esse tipo de percepção. As ações de responsabilidade social desenvolvidas pelas instituições financeiras podem contribuir para a melhoria da confiança nestas empresas, diminuindo também o ceticismo dos consumidores acerca dos motivos pelos quais estas empresas se engajam em ações sociais. (MATTILA et al., 2010)

Estudos de RSC são particularmente importantes para bancos de varejo devido, principalmente, a pouca diferenciação entre os serviços oferecidos por estas organizações (POOLTHONG; MANDHACHITARA, 2009; MANDHACHITARA; POOLTHONG, 2011), podendo assim, as ações de RSC tornarem-se um diferencial competitivo (OGRIZEK, 2002; LUO; BHATTACHARYA, 2006; PIVATO et al., 2008; SWAEN; CHUMPITAZ, 2008; MELO; GALAN, 2011; PELOZA; SHANG, 2011) que conduz a diversos benefícios. (LICHTENSTEIN et al., 2004; VLACHOS et al., 2009)

Em consonância com a visão estratégica da RSC, este trabalho tem por objetivo principal ampliar a compreensão da relação entre RSC e valor de marca por meio do entendimento da forma de influência exercida pela confiança do consumidor na empresa sobre a relação. Nesse sentido, foram propostos três modelos alternativos para verificar se a relação entre a RSC e o valor de marca ocorre diretamente (sem influência da confiança) ou, se esta relação é melhor compreendida quando a confiança do consumidor na empresa atua mediando (completa ou parcialmente) a relação entre os constructos. Os modelos e hipóteses de pesquisa foram testados a partir de dados coletados junto a clientes do Banco do Estado do Rio Grande do Sul (Banrisul), uma das maiores instituições financeiras do país.

Este estudo justifica-se, fundamentalmente, sobre quatro aspectos. O primeiro é a falta de consenso na literatura sobre a relação entre a RSC e os benefícios gerados para as empresas. Estudos empíricos têm demonstrado que esta relação pode ocorrer de forma direta ou pode ser mediada por outros constructos. O segundo é que a maior parte dos estudos, que tem relacionado a RSC com o valor da empresa, tem mensurado valor da empresa a partir de métricas financeiras ou contábeis, como preço de mercado, retorno das ações, retorno sobre ativo e retorno sobre patrimônio líquido (ORLITZKY et al., 2003). Neste trabalho, utiliza-se a mensuração do valor a partir da ótica do consumidor, por meio do valor de marca (YOO; DONTHU, 2001), representando uma ampliação do conhecimento no tema. O terceiro aspecto é que a maior parte dos estudos sobre RSC tem se concentrado em países desenvolvidos (MANDHACHITARA; POOLTHONG, 2011), no Brasil verifica-se que ainda há um grande espaço para exploração do tema (DAMEDA, 2012). Por fim, apesar da importância da RSC para o setor bancário, poucos estudos têm utilizado este campo empírico. (BENEKE et al., 2012)

\section{Referencial Teórico}

Esta seção apresenta e discute as variáveis que compõem este estudo - responsabilidade social corporativa, valor de marca e confiança - e, ainda, propõem as hipóteses de pesquisa a serem investigadas. 


\subsection{Responsabilidade Social Corporativa}

Apesar dos primeiros relatos de ações sociais desenvolvidas por organizações privadas remontarem ao século XVI, apenas na década de 1950 a RSC começou a ser conceituada de forma semelhante a que é utilizada na atualidade (REIS; SANTOS, 1996). O grande avanço da RSC ocorreu na década de 1960 nos Estados Unidos, tendo seus princípios básicos fundamentados na filantropia. (CARROLL, 1979)

No Brasil, a produção acadêmica sobre RSC aumentou de forma considerável a partir da década de 1990 (ANDRADE et al., 2010), devido, principalmente, à publicação dos primeiros balanços sociais por organizações privadas (TREVISAN, 2002). Apesar desse volume crescente, para Moretti e Campanário (2009), no Brasil, a RSC ainda se constitui em um campo de estudo em desenvolvimento e pouco maduro cientificamente.

As ações de RSC podem ser classificadas de diversas maneiras incluindo filantropia, marketing relacionado a causas, responsabilidade ambiental, forma de tratamento de funcionários, princípios éticos, legais, entre outras. (ELLEN et al., 2006; ANDRADE et al., 2010)

Para Peloza e Shang (2011), as atividades de RSC podem ser classificadas em três amplas categorias: filantropia, práticas de negócios ou relacionadas a produtos. Em uma revisão da literatura Peloza e Shang (2011) verificaram que, das práticas filantrópicas, as mais comumente estudadas são o marketing relacionado a causas, doações em dinheiro, envolvimento com a comunidade e voluntarismo de funcionários. $\mathrm{Na}$ categoria práticas de negócios destacam-se estudos que envolvem práticas de proteção ambiental, combate ao trabalho infantil, comportamento ético e diversidade. A categoria relacionada aos produtos inclui produtos orgânicos, verdes e produtos livres de resíduos.

Ogrizek (2002) considera que as ações de RSC escolhidas pelas empresas estão vinculadas ao contexto. O autor argumenta que nos Estados Unidos os esforços de RSC das instituições financeiras são centrados em projetos para as comunidades próximas e em práticas direcionadas aos recursos humanos. Já na Europa, as instituições financeiras têm incluído a RSC em sua filosofia empresarial e priorizado atividades de proteção ambiental e políticas de sustentabilidade.
A RSC é um constructo de difícil definição e que não possui consenso na literatura sobre a melhor forma de mensurá-lo (MELO; GALAN, 2011; PELOZA; SHANG, 2011). Em relação à mensuração da RSC, estudos têm empregado escalas uni ou multi dimensionais. Lichtenstein et al. (2004) por exemplo, desenvolveram uma escala unidimensional (cinco itens) que mensura a percepção dos consumidores de que as ações de RSC trazem retorno para a comunidade. Outros autores, como Andrade et al. (2010), têm se baseado no estudo de Carroll (1991) e optado por mensurar a RSC a partir das dimensões econômica, legal, ética e filantrópica.

\subsection{Valor de Marca}

Aaker (1998, p. 16) define valor de marca como o "[...] conjunto de passivos e ativos ligados a uma marca, seu nome e seu símbolo, que se somam ou se subtraem do valor proporcionado por um produto ou serviço para uma empresa e/ou para os consumidores dela".

Para Keller (1993), valor de marca representa o efeito diferencial do conhecimento da marca na resposta do consumidor às ações de marketing da marca. Valor de marca é considerado um ativo relacional que reside no mercado, pois não pertence à empresa, mas ocorre a partir do relacionamento dos consumidores com a marca da empresa (Ambler, 1997; Delgado-Ballester e Munuera-Alemán, 2005). Dessa forma, todas as ações de marketing produzem efeitos (positivos ou negativos), no longo prazo, sobre o valor de marca. (YOO; DONTHU, 2001)

Para Ambler (1997), o valor de marca pode ser utilizado como substituto para o desempenho organizacional no longo prazo, pois representa os hábitos e atitudes dos consumidores para com a marca representando um possível estoque futuro de lucros para a empresa.

\subsection{Responsabilidade Social Corporativa e Valor de Marca}

Hoeffler e Keller (2002) sugerem que ações sociais são um importante meio para as empresas construírem valor de marca. De acordo com os autores, ações de responsabilidade social podem melhorar a imagem 
corporativa, já que os consumidores podem associar empresas que executam ações sociais como sendo generosas e altruístas. Para Andrade et al. (2010), a $\mathrm{RSC}$ influenciaria positivamente o valor de marca, principalmente nas comunidades do entorno das empresas, já que, muitas vezes, grande parte do investimento em $\mathrm{RSC}$ é realizado em prol destas comunidades. Empiricamente, Melo e Galan (2011), verificaram a correlação significativa e positiva entre a RSC e valor de marca, apesar desta correlação ser de baixa intensidade. Andrade et al. (2010) destacam que, em seu estudo, o valor de marca foi influenciado positivamente pelas dimensões éticas e filantrópicas da RSC.

Dessa forma, sugere-se a primeira hipótese do estudo:

H1: A RSC influencia direta e positivamente o valor de marca da empresa.

\subsection{Confiança}

Castaldo et al. (2010) afirmam que o constructo confiança tem sido estudado por mais de 50 anos e que, durante este período, 96 diferentes definições foram identificadas. A maior parte dessas definições representa uma expectativa de que as ações futuras da parte confiada produzirão resultados positivos (ou pelo menos não negativos) na parte que confia.

A literatura apresenta três perspectivas distintas de confiança. A confiança racional ou calculista, baseada na conveniência econômica, em que a parte que confia acredita que terá os resultados esperados a partir da confiança na outra parte. A confiança cognitiva, fundamentada no conhecimento específico sobre o comportamento, competências e habilidades da outra parte. E a confiança, baseada em valores, que pressupõe que a confiança se desenvolve em estágios mais avançados do relacionamento, quando ocorre um grau crescente de interdependência entre as partes. (PIVATO et al., 2008)

Apesar do grande volume de estudos, entretanto, há divergências quanto à dimensionalidade do constructo confiança, sendo identificadas uma, duas ou até três dimensões. As dimensões encontradas nestes estudos indicam que a confiança na organização pode envolver aspectos de competência (capacidade de cumprir com as expectativas), honestidade (ma- nutenção de promessas) e benevolência. (SWAEN; CHUMPITAZ, 2008)

No setor bancário, a confiança é particularmente importante por indicar que a instituição possui credibilidade e competência para realizar seu serviço e trata seus clientes com benevolência. (POOLTHONG; MANDHACHITARA, 2009)

\subsection{Confiança, RSC e Valor de Marca}

Para Pivato et al. (2008), o resultado imediato das ações de RSC é o aumento da confiança na organização e em seus produtos por parte dos stakeholders. Ellen et al. (2006) afirmam que a RSC tem o potencial de fazer com que os consumidores confiem na empresa por acreditarem que ela é realmente preocupada com a sociedade e se sente moralmente obrigada a contribuir com sua melhoria.

Segundo Vlachos et al. (2009), poucos estudos exploram o impacto da RSC sobre a confiança do consumidor. Empiricamente, Pivato et al. (2008) e Du et al. (2011) verificaram que os resultados obtidos pelas ações de RSC são mediados pela confiança, sendo assim, as ações de RSC corporativa só seriam efetivas na medida em que aumentassem a confiança dos consumidores na organização.

Na visão de Pivato et al. (2008), empresas socialmente responsáveis são consideradas de confiança, pois apresentam transparência nas informações fornecidas à comunidade, o que faz com que essa comunidade tenha facilidade de monitorar as atividades da empresa, sendo improvável que ela proceda de forma oportunista.

O valor de marca é considerado um ativo relacional baseado no mercado, que se origina do relacionamento dos consumidores com as marcas (AMBLER, 1997; DELGADO-BALLESTER; MUNUERA-ALEMÁN, 2005). A confiança, por sua vez, é considerada o constructo-chave para a compreensão dos relacionamentos de longo prazo (MORGAN; HUNT, 1994). Para Ambler (1997), a quebra da confiança do consumidor, por exemplo, o aumento excessivo de preços, leva à diminuição do valor de marca.

Enquanto a hipótese de pesquisa $H 1$ considerava uma relação positiva e direta entre RSC e valor de marca, estudos empíricos sustentam que a confiança do consumidor na empresa atua como um mediador 
no processo que relaciona a RSC ao valor de marca. A partir dessa constatação, propõe-se a seguinte hipótese de pesquisa:

H2: A relação entre a RSC e o valor de marca é mediada pela confiança do consumidor na empresa.

Apesar de a proposta de mediação ser sustentada pela literatura, torna-se relevante compreender, caso essa mediação ocorra, se é de forma completa ou parcial. Com essa finalidade, são apresentadas duas hipóteses de pesquisa derivadas de $\mathrm{H} 2$ :

H2a: A relação entre a RSC e o valor de marca é completamente mediada pela confiança do consumidor na empresa.

H2b: A relação entre a RSC e o valor de marca é parcialmente mediada pela confiança do consumidor na empresa.

\section{MÉtodo}

Dada a falta de consenso sobre o impacto, direto ou mediado, da RSC sobre as respostas do consumidor em relação a essas ações, para este estudo, foram propostos três modelos apresentados a seguir (Figura 1). No primeiro modelo ("sem mediação"), a RSC impacta diretamente o valor de marca e a confiança (H1). No segundo modelo ("mediação completa" H2a), a confiança atua como um mediador completo na relação RSC - VM. No último modelo ("mediação parcial" - H2b), o impacto da RSC sobre o VM ocorre diretamente e indiretamente por meio da confiança.

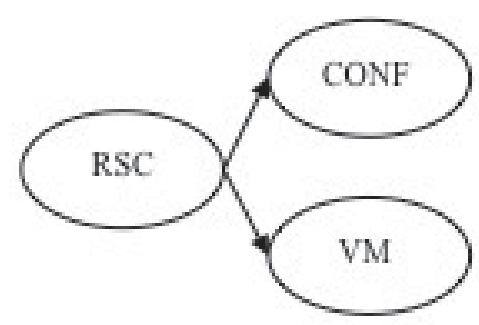

Modelo 1 ( $(\mathrm{sem}$ mediação)

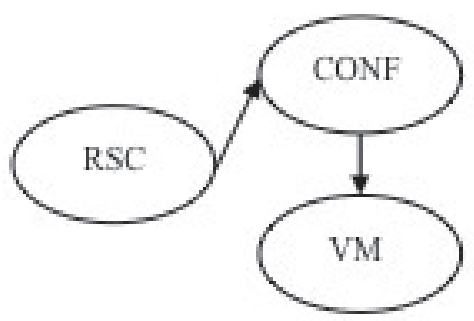

Modelo 2 (mediação completa)

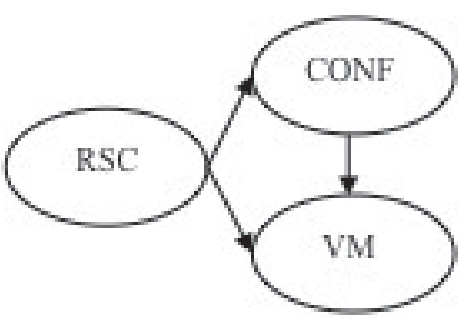

Modelo 3 (mediação parcial)

Figura 1: Modelos Propostos

Fonte: Elaborada pelos autores deste artigo

Os modelos e as hipóteses de pesquisa foram verificados por meio de modelagem de equações estruturais, conforme descrito na seção de resultados.

Para este estudo optou-se por buscar, junto à literatura, escalas já testadas empiricamente. Para o constructo responsabilidade social corporativa (RSC) utilizou-se a escala com cinco itens originalmente desenvolvida por Lichtenstein et al. (2004). Confiança foi mensurada por meio da escala de seis itens proposta por Aaker et al. (2004). Valor de marca foi mensurado por escala de quatro itens desenvolvida por Yoo $e$ Dunthu (2001) e validada no contexto nacional por Vargas Neto (2003).

As escalas de responsabilidade social corporativa e confiança foram traduzidas do inglês para o português. A adequação das escalas ao contexto do estudo foi feita por meio da aplicação do instrumento de pesquisa com cinco clientes da instituição estudada, selecionados por acessibilidade, que responderam ao questionário e o interpretaram, juntamente com um dos autores da pesquisa. Ocorreram somente pequenos ajustes de redação para adequação de linguagem $e$ facilitação de compreensão.

A coleta de dados, para teste dos modelos e das hipóteses de pesquisa, foi realizada em diversos locais públicos (amostra por conveniência - clientes da mesma instituição alvo do estudo); um total de 152 pessoas respondeu ao instrumento de pesquisa. A amostra final ficou reduzida a 148 respondentes devido a erros de preenchimento e excesso de dados faltantes (KLINE, 2011). Optou-se por não fazer pré-teste das escalas, pois elas já haviam sido validadas em outros contextos e, também, pela relativa facilidade de acesso aos respondentes. Os dados foram coletados no mês de maio 
de 2011 na cidade de Porto Alegre, RS. O questionário foi aplicado pessoalmente pelos próprios autores.

Os dados deste estudo foram coletados junto a clientes (pessoa física) do Banco do Estado do Rio Grande do Sul (Banrisul). O Banrisul foi escolhido por ser um dos maiores bancos nacionais em volume de ativos, possuir diversos programas sociais (18 no momento do estudo) e investir um volume substancial de recursos nesses programas.

O Banrisul foi criado em 1928 com o objetivo de fazer empréstimos de longo prazo. Atualmente o banco possui uma variada gama de serviços financeiros tanto para pessoas físicas quanto para pessoas jurídicas. Esses serviços são oferecidos em mais de 400 agências bancárias espalhadas por dez Estados brasileiros. No ano de 2011, o Banrisul possuía ativos superiores a $R \$$ 34 bilhões o que o colocava como o $12^{\circ}$ maior banco em atividade no país. (BANRISUL, 2012)

O Banrisul tem por política investir parte de sua receita em programas de responsabilidade social corporativa. A atuação social do banco concentra-se principalmente em educação, cultura, saúde, esporte e meio ambiente (BANRISUL, 2012). A partir do ano de 1999, o Banrisul passou a divulgar seu balanço social, especificando os programas sociais desenvolvidos, bem como os valores investidos. Na Tabela 1 é possível observar os valores investidos pelo Banrisul em ações de responsabilidade social corporativa entre os anos de 2005 e 2010.

Tabela 1: Investimento do Banrisul em ações de responsabilidade social corporativa 2005-2010

\begin{tabular}{|l|c|c|c|c|c|c|c|}
\hline & $\mathbf{2 0 0 5}$ & $\mathbf{2 0 0 6}$ & $\mathbf{2 0 0 7}$ & $\mathbf{2 0 0 8}$ & $\mathbf{2 0 0 9}$ & $\mathbf{2 0 1 0}$ \\
\hline Educação (em R\$ mil) & 2.590 & 1.813 & 1.011 & 1.571 & 5.330 & 728 \\
\hline Cultura (em R \$ mil) & 5.322 & 7.240 & 8.322 & 9.857 & 11.223 & 11.632 \\
\hline Saúde (em R \$ mil) & 72 & 170 & 65 & 33 & 8 & 112 \\
\hline Esporte (em R \$ mil) & 8.081 & 7.976 & 8.000 & 8.942 & 14.459 & 1.632 \\
\hline Outros* (em R $\$$ mil) & 7.925 & 5.938 & 4.924 & 9.869 & 11.303 & 7.663 \\
\hline Total (em R $\$$ mil) & 23.990 & 23.137 & 22.322 & 30.271 & 42.323 & 21.767 \\
\hline \%RO** & $4,39 \%$ & $4,21 \%$ & $3,57 \%$ & $4,30 \%$ & $4,96 \%$ & $1,90 \%$ \\
\hline \%RL*** & $1,38 \%$ & $1,22 \%$ & $1,03 \%$ & $1,39 \%$ & $1,88 \%$ & $0,88 \%$ \\
\hline
\end{tabular}

*Inclui atividades relacionadas ao meio ambiente **Receita operacional *** Receita líquida

Fonte: Elaborada pelos autores a partir de dados publicados pelo Banrisul (2012)

Destaca-se, na Tabela 1, que os investimentos em ações de RSC no ano de 2010 representaram, em valores absolutos, apenas metade daqueles verificados no ano de 2009. Em termos relativos (\%RO e \%RL), a queda dos investimentos sociais mostrou-se ainda mais acentuada, talvez motivados pela crise financeira mundial de 2008 e 2009.

\section{Análise dos Dados}

A amostra deste estudo apresentou predominância de respondentes do gênero feminino (87 respondentes $-58,78 \%$ da amostra), em sua maioria $(52,7 \%)$ possuíam entre 26 e 45 anos, $66,89 \%$ dos entrevistados tinham pelo menos nível superior incompleto e a faixa de renda predominante $(70,27 \%$ da amostra) situava-se entre $\mathrm{R} \$ 1.750,01$ e $\mathrm{R} \$ 3.000,00$. A Tabela 2 apresenta a caracterização completa da amostra do estudo.

A Tabela 3 (apesar de serem apresentadas neste ponto do texto, as informações sobre os programas sociais do Banrisul constavam no final do instrumento de coleta de dados de forma a não influenciarem as respostas às escalas de mensuração dos constructos) indica o grau de importância atribuído pelos consumidores às áreas de atuação social do Banrisul. O conhecimento e a importância dos programas sociais foram mensurados em escala de sete pontos. 
Tabela 2: Caracterização da amostra

\begin{tabular}{|c|c|c|c||c|c|}
\hline \multicolumn{2}{|c|}{ Faixa etária N (\%) } & \multicolumn{2}{c|}{ EscolaridadE* N (\%) } & \multicolumn{2}{c|}{ Renda FamiliaR N (\%) } \\
\hline 18 a 25 anos & $28(18,92 \%)$ & Ensino fundamental & $06(04,05 \%)$ & Até $\mathrm{R} \$ 1.750,00$ & $48(32,43 \%)$ \\
\hline 26 a 35 anos & $37(25,00 \%)$ & Ensino médio & $43(29,06 \%)$ & De $\mathrm{R} \$ 1.750,01$ a $\mathrm{R} \$ 3.000,00$ & $56(37,84 \%)$ \\
\hline 36 a 45 anos & $41(27,70 \%)$ & Ensino superior & $74(50,00 \%)$ & De $\mathrm{R} \$ 3.000,01$ a $\mathrm{R} \$ 7.000,00$ & $36(24,32 \%)$ \\
\hline 46 a 55 anos & $27(18,24 \%)$ & Pós graduação & $25(16,89 \%)$ & Acima de $\mathrm{R} \$ 7.000,01$ & $08(05,41 \%)$ \\
\hline 56 a 65 anos & $12(08,11 \%)$ & & & & \\
\hline Mais de 65 anos & $03(02,03 \%)$ & & & \\
\hline
\end{tabular}

*As classes de escolaridade incluem tanto o nível completo quanto incompleto

Fonte: Dados da pesquisa

Tabela 3: Grau de Importância dos Programas Sociais do Banrisul

\begin{tabular}{|c|c|c|}
\hline ÁreAs & MédIA DE IMPORTÂNCIA & DeSvio PADRÃo \\
\hline Educação & 6,47 & 1,32 \\
\hline Saúde & 6,47 & 1,31 \\
\hline Meio ambiente* & 6,01 & 1,50 \\
\hline Cultura & 5,92 & 1,51 \\
\hline Esporte & 5,16 & 1,99 \\
\hline
\end{tabular}

*Nos balanços sociais divulgados pelo Banrisul esta rubrica recebe a denominação de "outros"

Fonte: Dados da pesquisa

Tabela 4: Conhecimento e Importância dos Programas Sociais do Banrisul

\begin{tabular}{|c|c|c|c|c|}
\hline \multirow{2}{*}{$\begin{array}{l}\text { Programas } \\
\text { Crédito Leitura }\end{array}$} & \multicolumn{2}{|c|}{ CONHECIMENTO (\%) } & \multicolumn{2}{|c|}{ IMPORTÂNCIA (\%) } \\
\hline & Nenhum ou pouco & 89,86 & Alta & 66,67 \\
\hline Pescar & Nenhum ou pouco & 83,78 & Alta & 78,72 \\
\hline Martin Pescador & Nenhum ou pouco & 93,92 & Alta & 68,75 \\
\hline Orquestra de Câmara Jovem do RS & Nenhum ou pouco & 87,16 & Alta & 83,33 \\
\hline Concertos Banrisul para a Juventude & Nenhum ou pouco & 87,84 & Alta & 75,58 \\
\hline Karatê além do Esporte & Nenhum ou pouco & 94,59 & Alta & 90,00 \\
\hline Apoio a times de Futebol & Alto & 58,78 & Alta & 60,91 \\
\hline Crianças no Esporte & Nenhum ou pouco & 87,83 & Alta & 84,85 \\
\hline Mamamóvel & Nenhum ou pouco & 90,54 & Alta & 95,00 \\
\hline Controle Médico e Saúde Ocupacional & Nenhum ou pouco & 91,89 & Alta & 89,47 \\
\hline Ginástica Laboral & Nenhum ou pouco & 94,59 & Alta & 100,00 \\
\hline Banribike & Nenhum ou pouco & 97,30 & Alta & 77,78 \\
\hline Protocolo Verde & Nenhum ou pouco & 97,97 & Alta & 100,00 \\
\hline Reciclar & Nenhum ou pouco & 83,78 & Alta & 91,43 \\
\hline Sementes Banrisul & Nenhum ou pouco & 93,24 & Alta & 85,10 \\
\hline SOS Enchente & Nenhum ou pouco & 64,86 & Alta & 100,00 \\
\hline Campanha do Agasalho & Alto & 67,57 & Alta & 97,25 \\
\hline Voluntariado Banrisul & Nenhum ou pouco & 89,19 & Alta & 90,12 \\
\hline
\end{tabular}

Fonte: Dados da pesquisa 
Os dados da Tabela 3 evidenciam que os clientes do Banrisul consideram educação e saúde como as áreas de atuação social mais importantes, enquanto cultura e esporte seriam áreas menos prioritárias para ações sociais. Meio ambiente ficou em uma posição intermediária em relação às demais áreas. Nota-se que os resultados aqui apresentados não se alinham com as prioridades de investimentos sociais realizadas pelo Banrisul. No ano de 2010, as áreas que receberam maior investimento social foram respectivamente, cultura e meio ambiente, evidenciando uma falta de sintonia com a percepção dos respondentes.

A Tabela 4 apresenta o percentual de conhecimento e de importância atribuídos pelos clientes do Banrisul para cada um dos programas sociais específicos, desenvolvidos pela instituição.

Com exceção dos programas "apoio a times de futebol" e "campanha do agasalho", os demais programas sociais do Banrisul são praticamente desconhecidos pelos seus clientes. Pode-se atribuir o elevado conhecimento do programa "apoio a times de futebol" ao patrocínio que o banco faz aos dois times de futebol de maior torcida do Estado do Rio Grande do Sul. Já a campanha do agasalho é amplamente divulgada nos meio de comunicação. Em relação à importância atribuída pelos clientes aos programas sociais do Banrisul, nota-se que a pior avaliação foi sobre o programa "apoio a times de futebol" enquanto os programas "ginástica laboral", "protocolo verde" e "SOS enchente" foram considerados por todos os entrevistados como de importância alta. Esses resultados corroboram com os obtidos por Beneke et al. (2012) que verificaram que clientes possuem baixa consciência das ações de RSC desenvolvidas pelos bancos, assim um dos desafios para essas empresas é desenvolver estratégias de comunicação efetivas para suas ações de RSC.

Antes do teste de hipóteses propriamente dito, procedeu-se a análise da dimensionalidade e confiabilidade das escalas. Inicialmente a Análise Fatorial Exploratória (AFE) foi empregada para identificação da estrutura subjacente aos constructos (HAIR et al., 1995). A AFE foi necessária já que as escalas dos constructos ainda não haviam sido utilizadas em estudos nacionais e/ou no setor bancário. Na AFE, o método de extração empregado foi o de componentes principais com o critério de eingenvalues superiores a 1 para definição dos fatores. A confiabilidade das escalas foi verificada pelo Alfa de Cronbach. (CHURCHILL, 1979)

Os resultados da AFE revelaram soluções unidimensionais como mais adequadas para todos os constructos. O item "O Banrisul é socialmente responsável" da escala de RSC foi eliminado de procedimentos subsequentes, pois contribuía para elevada diminuição da confiabilidade da escala, além de representar um item genérico de avaliação, com caráter muito amplo. A escala de RSC ficou, então, definida por quatro itens que contribuíram para a maximização de sua confiabilidade $(\alpha=0,802)$. As escalas de confiança e valor de marca permaneceram com todos os itens originais e se mostraram adequadas para a mensuração dos respectivos constructos $(\alpha \mathrm{VM}=0,932$ e $\alpha \mathrm{CONF}=0,885)$ (no Apêndice 1 são apresentados os resultados da análise fatorial exploratória e confiabilidade das escalas).

Após a análise inicial (AFE e confiabilidade), os dados foram tratados por meio de modelagem de equações estruturais com a utilização do software Amos 18. A análise por modelagem de equações estruturais se dividiu em dois estágios: modelo de mensuração $e$ modelo estrutural.

O modelo de mensuração compreendeu a análise fatorial confirmatória (AFC) para a análise das cargas fatoriais dos indicadores dos constructos, avaliação da confiabilidade composta (CC), variância extraída (VE) e validade discriminante. Hair et al. (1995) recomendam que para um indicador seja mantido sua carga fatorial (padronizada) deve ser significativa e atingir pelo menos 0,5. Para CC e VE, Hair et al. (1995) sugerem, respectivamente, valores de iguais ou superiores a 0,6 e 0,5.

Pelos resultados da AFC, todos os itens das escalas de mensuração dos constructos apresentaram cargas fatoriais padronizadas significativas e superiores a 0,5 . A confiabilidade composta variou de 0,801 (RSC) a 0,937 (VM) enquanto a variância extraída de todas as escalas de mensuração situaram-se acima dos padrões recomendados por Hair et al. (1995) (os resultados da análise fatorial confirmatória, confiabilidade composta e variância extraída são apresentados no Apêndice 2).

A validade discriminante entre os constructos foi testada segundo a proposta de Fornell e Larcker (1981). Os autores recomendam que a variância extraída de cada construto deva ser superior à variância compartilhada (correlação ao quadrado) entre cons- 
trutos. O resultado do teste de validade discriminante demonstrou que os constructos representam fenômenos distintos - o Apêndice 3 apresenta o resultado do teste de validade discriminante conforme sugerido por Fornell e Larcker (1981). Conjuntamente, os resultados indicaram que as escalas de mensuração dos constructos eram adequadas para que se procedesse ao teste de hipóteses a partir dos modelos estruturais.

O teste de hipóteses foi realizado a partir da comparação dos modelos estruturais propostos (HAIR et al., 1995), sendo considerado superior o modelo melhor ajustado aos dados coletados. A estimação dos modelos foi por máxima verossimilhança, já que o pres- suposto desse método de estimação é a normalidade dos dados (BYRNE, 2010; KLINE, 2011). A normalidade dos dados foi observada em consonância com o critério de assimetria inferior a $|3|$ e curtose inferior a |10| (KLINE, 2011). Os dados coletados para todos os indicadores dos constructos ficaram dentro dos limites recomendados (assimetria entre $|0,145|$ e $|1,388|$, curtose entre $|0,029|$ e $|1,625|)$.

Como se pode observar na Tabela 5, os modelos estimados apresentaram alguns índices de ajuste fora dos parâmetros recomendados pela literatura. (HAIR et al., 1995)

Tabela 5: Índices de Ajuste Recomendados e Observados nos Modelos Propostos

\begin{tabular}{|c|c|c|c|c|}
\hline ÍNDICES & $\begin{array}{l}\text { VALORES RECOMENDA- } \\
\text { DOS* }\end{array}$ & $\begin{array}{c}\text { Modelo } 1 \\
\text { (SEM MEDIAÇão) }\end{array}$ & $\begin{array}{c}\text { Modelo } 2 \\
\text { (MEDIAÇÃo COMPLETA) }\end{array}$ & $\begin{array}{c}\text { MOdELO } 3 \\
\text { (MEDIAÇÃO PARCIAL) }\end{array}$ \\
\hline$\chi^{2}$ & - & 231,941 & 165,230 & 165,099 \\
\hline $\mathrm{DF}$ & - & 75 & 75 & 74 \\
\hline$\chi^{2} / \mathrm{DF}$ & $5 \leq$ & 3,093 & 2,203 & 2,231 \\
\hline GFI & $\geq 0,9$ & 0,841 & 0,872 & 0,872 \\
\hline CFI & $\geq 0,9$ & 0,888 & 0,936 & 0,935 \\
\hline TLI & $\geq 0,9$ & 0,864 & 0,922 & 0,920 \\
\hline RMSEA & 0,05 a 0,08 & 0,119 & 0,090 & 0,092 \\
\hline AIC & $* *$ & 291,941 & 225,230 & 227,099 \\
\hline ECVI & $* *$ & 1,986 & 1,532 & 1,545 \\
\hline PNFI & $* *$ & 0,696 & 0,733 & 0,723 \\
\hline
\end{tabular}

*Hair et al. (1995) ** Índices de comparação de modelos, segundo Hair et al. (1995) modelos com valores menores para AIC e ECVI possuem melhor ajuste, para PNFI valores maiores indicam melhor ajuste.

Fonte: Dados da pesquisa

A partir da análise dos índices de modificação (MI), sugeridos pelo software Amos, observou-se que a adição de parâmetros traria incremento ao ajuste dos modelos. Seguindo os valores em ordem decrescente dos índices de modificação, os parâmetros sugeridos foram adicionados um por vez e os modelos reestimados. O primeiro parâmetro adicionado foi entre os erros dos indicadores CONF5 e CONF6 (MI=20,786; Par change $=0,840$ ). Os indicadores envolvidos nesta modificação possuem relação quanto ao conteúdo; enquanto o indicador CONF5 considera a imagem da organização, o indicador CONF6 diz respeito às falhas dos serviços da empresa. Uma falha dos serviços possivelmente afetaria a imagem da organização, revelando a correlação entre esses indicadores. Dessa forma, conforme argumenta Byrne (2010), o índice de modificação pode ser utilizado já que possui argumento teórico que o sustente. O próximo parâmetro adicionado foi entre os erros dos indicadores RSC3 e RSC4 $(\mathrm{MI}=14,256 ;$ Par change $=0,583)$. Nesse caso, ambos os indicadores possuem conteúdo muito semelhantes, versando sobre investimentos sociais locais (RSC3) e apoio a atividades sociais importantes (RSC4). A estimação dos modelos sugeriu ainda que o parâmetro entre os erros de RSC1 e RSC4 deveria ser incluído para melhoria de ajuste $(\mathrm{MI}=10,534$; Par change $=0,424)$. O item RSC1 fala sobre investimento dos lucros em atividades sociais, já que apoio e investimento podem ser entendidos como sinônimos pelos respondentes; verifica-se a proximidade de conteúdo que suporta a 
inclusão de covariância entre os indicadores (Byrne, 2010). Após esta última alteração, todos os índices de modificação ficaram abaixo do valor 10 , indicando que nenhuma modificação traria melhorias de ajuste ao modelo (Byrne, 2010).

A Tabela 6 apresenta os índices de ajuste para os modelos com as alterações sugeridas pelos índices de modificação.

Nota-se que a adição de parâmetros trouxe melhorias de ajuste aos modelos propostos. Analisando os índices de ajuste dos modelos, conclui-se que o modelo 1 (sem mediação), mesmo com as modificações realizadas, continuou com índices de ajuste (GFI e RMSEA) fora dos parâmetros recomendados (HAIR et al., 1995). Dessa forma, este modelo não se mostrou adequado para o teste das relações propostas por ele, indicando não haver suporte para a hipótese H1. Em outras palavras, a relação entre RSC e valor de marca não é adequadamente explicada quando esses constructos relacionam-se diretamente.

Como esperado, os modelos 2 e 3 possuem índices de ajuste bastante próximos, já que a diferença entre os modelos é de apenas um parâmetro. A superioridade do modelo 2 (mediação completa) em relação ao modelo 3 (mediação parcial) revela-se pelos melhores índices de parcimônia (AIC, ECVI e PNFI) utilizados na comparação de modelos rivais (HAIR et al., 1995). A Tabela 7 apresenta os parâmetros estimados para os três modelos testados.

Tabela 6: Índices de ajuste recomendados e observados nos modelos propostos com as alterações sugeridas

\begin{tabular}{|c|c|c|c|c|}
\hline ÍNDICES & VALORES RECOMENDADOS* & $\begin{array}{c}\text { Modelo } 1 \\
\text { (SEM MEdIAÇão) }\end{array}$ & $\begin{array}{c}\text { MOdELO } 2 \\
\text { (MEDIAÇÃo COMPLETA) }\end{array}$ & $\begin{array}{c}\text { MODELO } 3 \\
\text { (MEDIAÇÃO PARCIAL) }\end{array}$ \\
\hline$\chi^{2} \mathrm{X}^{2}$ & - & 179,005 & 108,543 & 108,418 \\
\hline DF & - & 72 & 72 & 71 \\
\hline$\chi^{2} / \mathrm{DF}$ & $5 \leq$ & 2,486 & 1,508 & 1,527 \\
\hline GFI & $\geq 0,9$ & 0,875 & 0,912 & 0,912 \\
\hline CFI & $\geq 0,9$ & 0,924 & 0,974 & 0,973 \\
\hline TLI & $\geq 0,9$ & 0,903 & 0,967 & 0,966 \\
\hline RMSEA & 0,05 a 0,08 & 0,101 & 0,059 & 0,060 \\
\hline AIC & $* *$ & 245,005 & 174,543 & 176,418 \\
\hline ECVI & $* *$ & 1,667 & 1,187 & 1,200 \\
\hline PNFI & $* *$ & 0,696 & 0,734 & 0,724 \\
\hline
\end{tabular}

*Hair et al. (1995) ** Índices de comparação de modelos, segundo Hair et al. (1995) modelos com valores menores para AIC e ECVI possuem melhor ajuste, para PNFI valores maiores indicam melhor ajuste.

Fonte: Dados da pesquisa

Tabela 7: Parâmetros estimados para os modelos propostos

\begin{tabular}{|c|c|c|c|c|c|c|}
\hline \multirow[b]{2}{*}{ HIPÓTESES } & \multicolumn{2}{|c|}{$\begin{array}{c}\text { Modelo } 1 \\
\text { (SEM MEDIAÇÃo) }\end{array}$} & \multicolumn{2}{|c|}{$\begin{array}{c}\text { Modelo } 2 \\
\text { (MEDIAÇÃo COMPLETA) }\end{array}$} & \multicolumn{2}{|c|}{$\begin{array}{c}\text { ModELO } 3 \\
\text { (MEDIAÇÃo PARCIAL) }\end{array}$} \\
\hline & $\beta$ ( $\beta$ padr.) & $t$ & $\beta$ ( $\beta$ padr.) & $t$ & $\beta$ ( $\beta$ padr.) & $t$ \\
\hline $\mathrm{RSC} \rightarrow \mathrm{CONF}$ & $0,451(0,415)$ & 4,104 & $0,437(0,373)$ & 3,941 & $0,441(0,376)$ & 3,949 \\
\hline $\mathrm{RSC} \rightarrow \mathrm{VM}$ & $0,537(0,292)$ & $3,155^{*}$ & - & - & $-0,049(-0,026)$ & $-0,352 * *$ \\
\hline $\mathrm{CONF} \rightarrow \mathrm{VM}$ & - & - & $1,142(0,705)$ & 8,266 & $1,159(0,716)$ & 7,882 \\
\hline $\mathrm{R}^{2} \mathrm{CONF}$ & 0,172 & & 0,139 & & 0,141 & \\
\hline $\mathrm{R}^{2} \mathrm{VM}$ & 0,085 & & 0,497 & & 0,499 & \\
\hline
\end{tabular}

$\mathrm{p}=0,000 ; * \mathrm{p}=0,002 ; * * \mathrm{p}=0,725$ (não significativo)

Fonte: Dados da pesquisa 
Verifica-se que, o único parâmetro adicional do modelo $3(\mathrm{RSC} \rightarrow \mathrm{VM})$ em relação ao modelo 2 , não apresentou significância estatística, e o sinal da carga foi negativo, o que não encontra respaldo teórico. Essa constatação reforça que a confiança media completamente a relação entre a RSC e o valor de marca.

Verifica-se, ainda, que no modelo de mediação completa (modelo 2), a RSC (indiretamente por meio da confiança) e a confiança contribuíram para explicação de aproximadamente $50 \%$ da variância do valor de marca $\left(R^{2} V M=0,497\right)$. Apenas como forma de comparação, observa-se que no modelo sem mediação (modelo 1), a RSC permitiu explicar apenas 8,5\% da variância do valor de marca.

Assim, os resultados apresentados dão suporte às hipóteses $\mathrm{H} 2$ e H2a, ou seja, verifica-se que a relação entre a RSC e o valor de marca é mediada pela confiança do consumidor na empresa $(H 2)$ e que essa mediação ocorre de forma completa $(H 2 a)$.

\section{Conclusões, Implicações Práticas e Limitações e Sugestões de Estudos FUTUROS}

A seguir são apresentadas as conclusões da pesquisa com foco nas implicações teóricas e práticas, assim como as limitações e sugestões de estudos futuros.

\subsection{Conclusões}

O objetivo principal deste trabalho foi analisar o papel da confiança na relação entre a RSC e o valor de marca. Para atingir esse objetivo foram testados empiricamente três modelos relacionando os constructos. O primeiro modelo relacionava diretamente a RSC com a confiança e o valor de marca. O segundo modelo considerava que a confiança mediava completamente a relação entre a RSC e o valor de marca. Já, no último modelo, a RSC impactava indiretamente, por meio da confiança, e diretamente o valor de marca (mediação parcial).

A comparação entre os modelos revelou a inferioridade do modelo 1 (sem mediação) em relação aos demais, já que ele possuía alguns índices de ajuste fora dos parâmetros recomendados pela literatura. Já em relação aos modelos que propunham a confiança como mediador, o modelo com mediação completa demonstrou-se superior, principalmente em função dos índices de parcimônia (AIC, ECVI e PNFI).

A partir dos resultados da comparação dos modelos, procedeu-se a verificação das hipóteses de pesquisa. A hipótese $(H 1)$ que previa relação direta $e$ positiva entre RSC e valor de marca não foi suportada. Já a relação entre RSC e valor de marca, mediada pela confiança foi suportada (H2), sendo explicada de melhor forma por uma mediação completa $(H 2 a)$.

Concluiu-se, assim, que os resultados não demonstraram suporte para uma relação direta entre RSC e valor de marca, entretanto, ao incluir a mediação completa dessa relação pela confiança, praticamente $50 \%$ do valor de marca passa a ser explicado.

Os resultados deste estudo corroboram com a vertente da literatura (PIVATO et al., 2008; SWAEN; CHUMPITAZ, 2008; VLACHOS et al., 2009; POOLTHONG; MANDHACHITARA, 2009; STANALAND et al., 2011) que defende o papel mediador da confiança na relação entre as ações de RSC e os resultados obtidos pelas empresas.

As hipóteses suportadas indicam que as práticas de RSC contribuem para o aumento da confiança dos clientes na instituição e esta, por sua vez, impacta positivamente o valor de marca. Desse modo, a melhoria da percepção dos clientes sobre a responsabilidade social de uma organização pode se reverter (indiretamente por meio da confiança na empresa) em melhoria do valor de marca.

O estudo colabora na ampliação do conhecimento sobre o tema RSC e suas consequências ao identificar benefícios sob a ótica do consumidor e ao utilizar dimensões mediadoras dessa relação, nesse caso, a confiança do consumidor na organização.

\subsection{Implicações Práticas}

Apesar de o estudo estar circunscrito a clientes de uma única instituição, os resultados podem trazer contribuições práticas para qualquer organização, especialmente àquelas do setor financeiro (bancos). Especificamente, os resultados deste estudo trazem dois tipos de implicações.

O primeiro é derivado das percepções dos respondentes sobre as questões gerais de relações entre RSC, valor de marca e confiança. As hipóteses permitem 
propor que a estratégia de investir em RSC traz, por intermédio do aumento da confiança do cliente na empresa, impactos positivos sobre o valor da marca. Esse ponto torna-se relevante como uma possível fonte de vantagem competitiva, especialmente em instituições financeiras, cuja diferenciação dos serviços é baixa. (POOLTHONG; MANDHACHITARA, 2009; MANDHACHITARA; POOLTHONG, 2011)

O segundo aspecto é derivado das respostas sobre as ações de RSC realizadas pela instituição pesquisada. Observa-se que, apesar do acerto em investir em RSC (como estratégia), há falhas na operacionalização das ações.

Os dados evidenciaram que os respondentes/ clientes creditam elevada importância para ações de RSC e dentre as diferentes categorias de ações valorizam mais aquelas ligadas à educação, à saúde e ao meio ambiente. Entretanto, os dados também evidenciaram que as ações da instituição pesquisada não estavam prioritariamente ligadas a essas áreas, o banco investe mais em ações ligadas a esporte e cultura. Ainda, ligado às ações realizadas pela instituição, os resultados demonstraram que os clientes têm um baixo conhecimento sobre elas.

Esses pontos chamam a atenção para a necessidade de um alinhamento das estratégias de RSC com a percepção dos clientes.

Sugere-se que, como forma de impactar a confiança e o valor de marca, a empresa priorize ações de $\mathrm{RSC}$ de acordo com categorias de ações valorizadas pelos clientes, no caso do Banrisul, educação, saúde e meio ambiente. Peloza e Shang (2011) alertam que, para serem efetivas, as atividades de RSC devem ser focadas naquelas que entregam mais valor ao grupo que se pretende atingir com a atividade (clientes, acionistas, público interno, etc.).

Um ponto a ser considerado é o envolvimento dos consumidores na definição das ações a serem realizadas. Estudos empíricos (SWAEN; CHUMPITAZ, 2008; DU et al., 2011) indicam que o envolvimento pessoal do consumidor nas ações de RSC se traduzem em aumento de confiança na empresa que é maior do que a verificada em consumidores que têm apenas consciência das ações sociais desenvolvidas pela organização.

Igualmente importante é a comunicação ou disseminação das ações realizadas junto aos clientes.
O desconhecimento, por parte dos clientes, sobre as ações de RSC realizados pela empresa pode ser visto como um recurso que não atinge plenamente seu potencial. Conforme Swaen e Chumpitaz (2008) e Du et al. (2010), para que as ações de RSC tragam ganhos para a organização, é necessário que a comunicação dessas ações tenham o poder de convencer os stakeholders que os motivos que guiam a organização são bem intencionados e não guiados puramente por questões econômicas. Beneke et al. (2012) consideram que, inicialmente, os consumidores precisam tornar-se conscientes das iniciativas sociais da empresa; o aumento da consciência levaria ao conhecimento sobre a empresa, o que resultaria em atitudes mais favoráveis para com ela em função de seu comprometimento com ações sociais.

\subsection{Limitações e Sugestões de Estudos Futuros}

A principal limitação deste estudo é a amostra. Este estudo utilizou como amostra clientes de apenas um banco, dificultando as generalizações. O tamanho da amostra também pode ser um problema já que a técnica de modelagem de equações estruturais requer amostras consideradas grandes, que na maior parte dos estudos superam os 200 casos. (BYRNE, 2010)

Pode-se considerar a melhoria das escalas ou utilização de outras escalas para os constructos responsabilidade social corporativa e confiança. O modelo de mensuração necessitou algumas modificações para atingir ajuste satisfatório; a análise dos índices de modificação (MI) e conteúdo dos indicadores são indícios de que pode ter havido colinearidade entre indicadores.

Como estudos futuros recomendam-se:

a) estudar como a comunicação das atividades de caráter social da empresa afeta seu valor de marca.

b) Inclusão no modelo proposto de outros construtos, além do valor de marca, que possam avaliar os benefícios da RSC, como intenção de permanência, comunicação boca a boca, entre outros.

Igualmente, o comprometimento e/ou envolvimento com causas sociais por parte do consumidor podem ser considerados como variáveis moderadoras do modelo, permitindo a segmentação dos clientes com 
base nestes constructos. Consumidores que são mais envolvidos com causas sociais são mais informados sobre as ações das empresas nesse sentido e, possivelmente, guiarão seu comportamento para com essas empresas com base no conhecimento que possuem sobre tais empresas e suas ações sociais. (GOLOB et al., 2008)

\section{REFERÊNCIAS}

AAKER, D. A. Brand equity: gerenciando o valor de marca. São Paulo: Negócio, 1998.

AMBLER, T. How much of brand equity is explained by trust? Management Decisions, Bingley, UK, v. 35, n. 4, p. 283-292, 1997.

ANDRADE, M. A. M. de; GOSLING, M.; JORDÃO, R. V. D. Valor de marca e responsabilidade social em siderúrgicas mineiras e a percepção de suas comunidades de entorno. In: XXXIV ENANPAD, Rio de Janeiro, ANPAD, 2010. Anais..., ANPAD, Rio de Janeiro, 2010.

BANRISUL. Balanço social. [2012]. Disponível em: <http://www.banrisul.com.br/bob/link/bobw00hn_ balanco_social.aspx?secao_id=25>. Acesso em: 20 mar. 2012.

BANRISUL. História. [2013]. Disponível em: < http:// www.banrisul.com.br/bob/link/bobw00hn_historia. aspx?secao_id $=23>$. Acesso em: $1^{\circ}$ abr. 2013.

BENEKE, J. Don't bank on it: delineating the relationship between corporate social responsibility and retail bank affinity. South African Journal of Business Management, Stelenbosch, South Africa, v. 43, n. 1, p. 45-54, 2012.

BYRNE, B. M. Structural equation modeling with AMOS - basic concepts, applications, and programming. 2. ed. New York: Routledge, 2010.

CARROLL, A. B. A three Dimensional Conceptual Model of Corporate Performance. Academy of Management Review, New York, USA, v. 4, n. 4, p. 497-505, 1979.
CARROLL, A. B. The pyramid of corporate social responsibility: toward the moral management of organizational stakeholder. Business Horizons, Indianapolis, USA, v. 34, n. 4, p. 39-48, 1991.

CASTALDO, S.; PREMAZZI, K.; ZERBINI, F. The meaning (s) of trust. A content analysis of the diverse conceptualizations of trust in scholarly research on business relationships. Journal of Business Ethics, New York, USA, v. 96, n. 4, p. 657-668, 2010.

CHURCHILL, G. A. Jr. A paradigm for developing better measures of marketing constructs. Journal of Marketing Research, Chicago, USA, v. 16, n. 1, p. 64-73, 1979.

DAMEDA, A. das N. Responsabilidade social corporativa (RSC): uma análise das tendências de pesquisa e abordagens metodológicas. In: XV SEMEAD SEMINÁRIOS EM ADMINISTRAÇÃO. São Paulo, USP, 2012, Anais... São Paulo, 2012.

DELGADO-BALLESTER, E.; MUNUERA-ALEMÁN, J.

L. Does brand trust matter to brand equity? Journal of

Product \& Brand Management, Bingley, UK, v. 14, n. 3, p. 187-196, 2005.

DU, S.; BHATTACHARYA, C. B.; SEN, S. Maximizing business returns tocorporate social responsibility (CSR): the role of CSR communication. International Journal of Management Reviews, London, UK, v. 12, n. 1, p. 8-19, 2010.

DU, S.; BHATTACHARYA, C. B.; SEN, S. Corporate social responsibility and competitive advantage: overcoming the trust barrier. Management Science, Catonsville, USA, v. 57, n. 9, p. 1.528-1.545, 2011.

ELLEN, P. S.; WEBB, D. J.; MOHR, L. A. Building corporate associations: consumer attributions for corporate socially responsible programs. Journal of Academy of Marketing Science, New York, USA, v. 34, n. 2, p. 147-157, 2006.

FORNELL, C.; LARCKER, D. Evaluating structural equation models with unobservable variables with measurement error. Journal of Marketing Research, Chicago, USA v. 18, n. 1, p. 39-50, 1981. 
GOLOB, U.; LAH, M.; JANČIČ, Z. Value orientations and consumer expectations of corporate social responsibility.

Journal of Marketing Comunications, London, UK, v. 14, n. 2, p. 83-96, 2008.

HAIR, J. F. et al. Multivariate data analysis with readings. 4. ed. Upper Saddle River: Prentice Hall, 1995.

HOEFFLER, S.; KELLER, K. L. Building brand equity through corporate societal marketing. Journal of Public Policy \& Marketing, New York, USA, v. 21, n. 1, p. 7889, 2002.

LICHTENSTEIN, D. R.; DRUMWRIGHT, M. E.; BRAIG, B. M. The effect of corporate social responsibility on customer donations to corporate-supported nonprofits. Journal of Marketing, Chicago, USA v. 68, n. 4, p. 1632,2004 .

LUO, X.; BHATTACHARYA, C. B. Corporate social responsibility, customer satisfaction, and market value. Journal of Marketing, Chicago, USA, v. 70, n. 4, p. 1-18, 2006.

KELLER, L. Conceptualizing, measuring, and managing customer-based brand equity. Journal of Marketing, Bingley, UK, v. 57, n. 1, p. 1-22, 1993.

KLINE, R. B. Principles and practice of structural equation modeling. 3. ed. New York: The Guilford Press, 2011.

MANDHACHITARA, R.; POOLTHONG, Y. A model of consumer loyalty and corporate social responsibility.

Journal of Services Marketing, Bingley, UK, v. 25, n. 2, p. 122-133, 2011.

MATTILA, A. S.; HANKS, L.; KIM, E. E. K. The impact of company type and corporate social responsibility messaging on consumer perceptions. Journal of Financial Services Marketing, London, UK, v. 15, n. 2, p. 126-135, 2010.

MELO, T.; GALAN, J. I. Effects of corporate social responsibility on brand value. Journal of Brand Managment, v. 18, n. 6, p. 423-437, 2011.
MORETTI, S. L. do A.; CAMPANÁRIO, M. de A. A produção intelectual brasileira em responsabilidade social empresarial - RSE sob a ótica da bibliometria. Revista de Administração Contemporânea, Rio de Janeiro, v. 13 (Edição Especial), p. 68-86, 2009.

MORGAN, R. M.; HUNT, S. D. The commitment-trust theory of relationship marketing. Journal of Marketing, Chicago, USA, v. 58, n. 3, p. 20-38, 1994.

NAN, X.; HEO, K. Consumer responses to corporate social responsibility (CSR) initiatives. Journal of Advertising, London, UK, v. 36, n. 2, p. 63-74, 2007.

NILSSON, J. Segmenting socially responsible mutual fund investors - the influence of financial return and social responsibility. International Journal of Bank Marketing, Bingley, UK, v. 27, n. 1, p. 5-31, 2009.

OGRIZEK, M. The effect of corporate social responsibility on the brading of financial services. Journal of Financial Services Marketing, London, UK,v. 6, n. 3, p. 215-228, 2002.

ORLITZKY, M.; SCHMIDT, F. L.; RYNES, S. L. Corporate social and financial performance: a meta-analysis.

Organizational Studies, Thousand Oaks, CA, v. 24, n. 3, p. 403-441, 2003.

PELOZA, J.; SHANG, J. How can corporate social responsibility activities create value for stakeholders? A systematic review. Journal of Academy of Marketing Science, New York, USA, v. 39, n. 1, p. 117-135. 2011.

PIVATO, S.; MISANI, N.; TENCATI, A. The impact of corporate social responsibility on consumer trust: the case of organic food. Business Ethics: a European Review, Oxford, UK, v. 17, n. 1, p. 3-12, 2008.

POOLTHONG, Y.; MANDHACHITARA, R. Customer expectations of CSR, perceived service quality and brand effect in Thai retail banking. International Journal of Bank Marketing, Bingley, UK, v. 27, n. 6, p. 408-427, 2009.

REIS, A. C. F; SANTOS, R. da C. Patrocínio e Mecenato: ferramenta de enorme potencial para as empresas.

Revista de Administração de Empresas, São Paulo, v. 36, n. 2, p. 17-25, 1996. 
STANALAND, A. J. S.; LWIN, M. O.; MURPHY, P.

E. Consumer perceptions of the antecedents and consequences of corporate social responsibility. Journal of Business Ethics, New York, USA, v. 102, n. 1, p. 47-55, 2011.

SWAEN, V.; CHUMPITAZ, R. C. Impact of corporate social responsibility on consumer trust. Recherche et Applications en Marketing, Paris, FR, v. 23, n. 4, p. 7-33, 2008.

TREVISAN, F. Balanço Social como Instrumento de Marketing. Revista Administração de Empresas, [online], v. 1, n. 2, p. 1-12, 2002.

\section{VARGAS NETO, A. Mensuração de Brand Equity}

baseada no consumidor: avaliação de escala multidimensional. 97p. Dissertação de Mestrado. UFGRS. Porto Alegre, RS, 2003.

VLACHOS, P. A.; TSAMAKOS, A.; VRECHOPOULOS, A. P.; AVRAMIDIS, P. K. Corporate social responsibility: attribuitions, loyalty, and the mediating role of trust. Journal of Academy of Marketing Science, New York, USA, v. 37, n. 2, p. 170-180, 2009.

YOO, B.; DONTHU, N. Developing and validating a multidimensional consumer-based brand equity scale. Journal of Business Research, Philadelphia, USA, v. 52, n. 1, p. 1-14, 2001. 


\section{Apêndice A - Média, Desvio Padrão, Carga Fatorial (CF), Variância Explicada (VE) e Alfa de Cronbach ( $\alpha$ ) das Escalas de Mensuração}

\begin{tabular}{|c|c|c|c|c|}
\hline $\begin{array}{c}\text { ReSPONSABILIDAde Social Corporativa - RSC } \\
\left(\mathrm{KMO}=\mathbf{0 , 6 1 6} \text {; MSA: }\left(\chi^{2}=\mathbf{2 1 8}, \mathbf{5 8 5} ; \text { DF }=\mathbf{6} ; \mathrm{P}<\mathbf{0 , 0 0 1}\right)\right.\end{array}$ & $\begin{array}{c}\text { MÉDIA } \\
\text { (DESVIO PADRÃo) }\end{array}$ & CF & VE & $\alpha$ \\
\hline RSC2 - O Banrisul retribui socialmente para as comunidades em que é atuante & $4,18(1,76)$ & 0,837 & \multirow{4}{*}{$62,83 \%$} & \multirow{4}{*}{0,802} \\
\hline RSC3 - Entidades sociais locais se beneficiam do apoio do Banrisul & $4,46(1,78)$ & 0,803 & & \\
\hline RSC1 - O Banrisul investe uma parcela dos lucros em organizações sociais & $4,41(1,69)$ & 0,793 & & \\
\hline RSC4 - O Banrisul apoia atividades sociais importantes & $4,71(1,57)$ & 0,733 & & \\
\hline \multicolumn{5}{|l|}{ 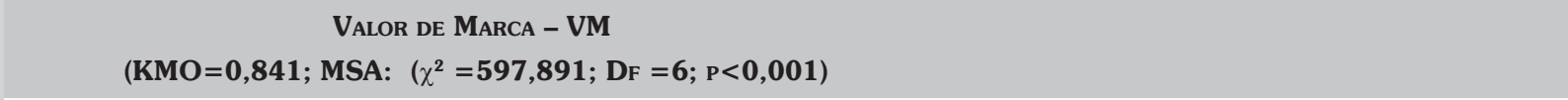 } \\
\hline $\begin{array}{l}\text { VM3 - Mesmo que exista outro banco tão bom quanto } \\
\text { o Banrisul, eu prefiro ser cliente do Banrisul }\end{array}$ & $4,84(2,40)$ & 0,957 & \multirow{4}{*}{$83,50 \%$} & \multirow{4}{*}{0,932} \\
\hline $\begin{array}{l}\text { VM2 - Mesmo que outro banco tenha as mesmas características } \\
\text { do Banrisul eu prefiro ser cliente Banrisul }\end{array}$ & $5,07(2,31)$ & 0,948 & & \\
\hline $\begin{array}{l}\text { VM1 - Eu prefiro ser cliente do Banrisul, em vez de qualquer } \\
\text { outro banco, mesmo que eles ofereçam o mesmo serviço }\end{array}$ & $4,93(2,39)$ & 0,943 & & \\
\hline $\begin{array}{l}\text { VM4 - Se os outros bancos não são diferentes do Banrisul de } \\
\text { nenhuma maneira, parece mais inteligente ser cliente Banrisul }\end{array}$ & $4,82(2,35)$ & 0,798 & & \\
\hline \multicolumn{5}{|l|}{$\begin{array}{c}\text { CONFIANÇA - CONF } \\
(\mathrm{KMO}=\mathbf{0 , 8 7 2} \text {; MSA: }(\chi 2=460,896 ; \mathrm{DF}=15 ; \mathrm{P}<0,001)\end{array}$} \\
\hline CONF3 - Eu tenho certeza que o Banrisul é responsável nas suas ações & $5,39(1,67)$ & 0,860 & \multirow{6}{*}{$64,56 \%$} & \multirow{6}{*}{0,885} \\
\hline CONF4 - O Banrisul é confiável & $5,84(1,48)$ & 0,815 & & \\
\hline $\begin{array}{l}\text { CONF5 - Dada à imagem que tenho do Banrisul, seria } \\
\text { surpresa que o banco me deixasse mal }\end{array}$ & $5,20(2,00)$ & 0,796 & & \\
\hline CONF1 - Eu posso sempre contar com o Banrisul para fazer o que é melhor & $4,95(1,85)$ & 0,786 & & \\
\hline $\begin{array}{l}\text { CONF2 - Se o Banrisul comete um erro, sei que ele } \\
\text { vai fazer o melhor para compensar isso }\end{array}$ & $4,81(1,94)$ & 0,782 & & \\
\hline $\begin{array}{l}\text { CONF6 - Uma falha de serviços do Banrisul seria } \\
\text { incompatível com as minhas expectativas }\end{array}$ & $5,10(2,06)$ & 0,778 & & \\
\hline
\end{tabular}

Fonte: Dados da pesquisa 


\section{Apêndice B - Carga Fatorial Padronizada, $T$, Confiabilidade Composta (CC) E VARIÂNCIA Extraída (VE)}

\begin{tabular}{|c|c|c|}
\hline Item - Constructo & CARGa FATORIAL PADRONIZADA & $T$ \\
\hline RSC1 - RSC & 0,764 & \\
\hline RSC2 - RSC & 0,847 & 9,009 \\
\hline RSC3 - RSC & 0,658 & 6,856 \\
\hline RSC4 - RSC & 0,547 & 5,848 \\
\hline $\mathrm{CC}$ & 0,801 & \\
\hline VE & 0,508 & \\
\hline VM1 - VM & 0,944 & \\
\hline VM2 - VM & 0,937 & 22,770 \\
\hline VM3 - VM & 0,957 & 24,649 \\
\hline VM4 - VM & 0,694 & 10,788 \\
\hline $\mathrm{CC}$ & 0,937 & \\
\hline VE & 0,792 & \\
\hline CONF1 - CONF & 0,724 & \\
\hline CONF2 - CONF & 0,732 & 8,529 \\
\hline CONF3 - CONF & 0,854 & 9,626 \\
\hline CONF4 - CONF & 0,795 & 9,101 \\
\hline CONF5 - CONF & 0,731 & 8,473 \\
\hline CONF6 - CONF & 0,704 & 8,148 \\
\hline $\mathrm{CC}$ & 0,890 & \\
\hline VE & 0,575 & \\
\hline
\end{tabular}

Fonte: Dados da pesquisa

\section{Apêndice C - Teste de validade discriminante de Fornell e Larcker (1981)*}

\begin{tabular}{|c|c|c|c|}
\hline \multicolumn{1}{|c|}{ RSC } & VM & CONF \\
\hline RSC & 0,508 & & \\
\hline VM & 0,077 & 0,792 \\
\hline CONF & 0,183 & 0,493 & 0,575 \\
\hline
\end{tabular}

*Variância extraída na diagonal e variância compartilhada fora da diagonal

Fonte: Dados da pesquisa 